\title{
A two-stage search strategy for detecting multiple loci associated with rheumatoid arthritis Pritam Chanda*1, Aidong Zhang ${ }^{1}$, Lara Sucheston ${ }^{2}$ and Murali Ramanathan ${ }^{3}$
}

\author{
Addresses: ${ }^{1}$ Departments of Computer Science and Engineering, State University of New York, Buffalo, New York 14260, USA, \\ ${ }^{2}$ Department of Biostatistics, State University of New York, Buffalo, New York 14260, USA and ${ }^{3}$ Department of Pharmaceutical Sciences, \\ State University of New York, Buffalo, New York 14260, USA \\ E-mail: Pritam Chanda* - pchanda@cse.buffalo.edu; Aidong Zhang - azhang@cse.buffalo.edu; Lara Sucheston - lsuchest@buffalo.edu; \\ Murali Ramanathan - murali@buffalo.edu \\ *Corresponding author
}

from Genetic Analysis Workshop 16

St Louis, MO, USA 17-20 September 2009

Published: 15 December 2009

BMC Proceedings 2009, 3(Suppl 7):S72 doi: 10.1186/1753-656I-3-S7-S72

This article is available from: http://www.biomedcentral.com/I753-656I/3/S7/S72

(C) 2009 Chanda et al; licensee BioMed Central Ltd.

This is an open access article distributed under the terms of the Creative Commons Attribution License (http://creativecommons.org/licenses/by/2.0), which permits unrestricted use, distribution, and reproduction in any medium, provided the original work is properly cited.

\section{Background}

The risk of developing many common and complex diseases such as cancer and autoimmune disease involve complex interactions between multiple genes and several endogenous and exogenous environmental factors (or covariates). Rheumatoid arthritis (RA) is a complex genetic disease in which it is hypothesized that several loci contribute to disease susceptibility. Information theoretic methods are among the most promising approaches for genetic association studies and have been used for genetic analysis $[1,2]$ and analysis of gene $x$ gene interactions $[3,4]$. In this paper, we describe our use of a two-stage strategy consisting of an information theoretic search followed by logistic regression to detect gene $\times$ gene interactions associated with RA using selected genomic regions from the genome-wide scan data from the North American Rheumatoid Arthritis Consortium, which comprises 868 cases and 1194 controls. Data were provided as Problem 1 of Genetic Analysis Workshop 16.

\section{Methods}

Interaction information as measure of association

Let $X_{i}$ denotes a genetic random variable representing the genotypes at locus $L_{i}$. We assume $L_{i}$ is biallelic (with alleles $A$ and $a$ ) with three possible genotypes $(A A, A a$, and $a a)$. The uncertainty of $X_{i}$ is given by Shannon's entropy [5] as 


$$
H\left(X_{i}\right)=-\sum_{x \in\{A A, A a, a a\}} P\left(X_{i}=x\right) \log _{2} P\left(X_{i}=x\right) .
$$

Given a set of such genetic variables $S=\left\{X_{1} ; X_{2} ; \ldots ; X_{k}\right\}$, the interaction information among the $k$ variables (referred to as $k$-way interaction information or KWII) is defined as the amount of information (redundancy or synergy) present in the set of variables that is not present in any subset of these variables [4]. For the variables in set $S$, the KWII can be written succinctly as an alternating sum over entropies $(H)$ of all possible subsets $\tau$ of $S$ using the difference operator [6]:

$$
K W I I(S) \equiv-\sum_{\tau \subseteq S}(-1)^{|S|-|\tau|} H(\tau) .
$$

Let $C$ be the random variable representing the disease status (phenotype variable) of $R A$. Then $\operatorname{KWII}(S ; C)=K W I I$ $\left(X_{1} ; X_{2} ; \ldots ; X_{k} ; C\right)$ is a measure of the association of the set of genetic variables in set $S$ towards the disease phenotype variable $C$ (i.e., how well the set explains the disease phenotype). The value of $\operatorname{KWII}(S ; C)$ can be both positive and negative. We shall use only positive KWII values as the measure of association because larger positive values indicate stronger interaction (hence, higher association).

\section{Redundancy between combinations of variables}

Let $S_{1}=\left\{X_{1} ; \ldots ; X_{m}\right\}$ and $S_{2}=\left\{Y_{1} ; \ldots ; Y_{m}\right\}$ be two sets (or combinations) of variables. Then the redundancy between $S_{1}$ and $S_{2}$ is given by the maximized average of pairwise linkage disequilibrium (LD) $\left(r^{2}\right)$ between variables from $S_{1}$ and $S_{2}$ :

$$
\operatorname{red}\left(S_{1}, S_{2}\right)=\max \left(\left(\sum_{X_{i} \in S_{1}, Y_{j} \in S_{2}} r^{2}\left(X_{i}, Y_{j}\right)\right) / m\right) .
$$

Such redundancies can arise because of LD between the variables across each set. For example, for a disease $C$ that is caused by interactions between two untyped SNPs $D_{1}$ and $D_{2}$, let four marker loci be designated $X_{1}, X_{2}, X_{3}$, and $X_{4}$ such that $X_{1}$ and $X_{3}$ are in strong $\mathrm{LD}$ with $D_{1}$, while $X_{2}$ and $X_{4}$ are in strong LD with $D_{2}$. Then the $\operatorname{KWII}\left(X_{1} ; X_{2} ; \mathrm{C}\right)$ and $\operatorname{KWII}\left(X_{3} ; X_{4} ; \mathrm{C}\right)$ measure the association of the sets $\left\{X_{1} ; X_{2}\right\}$ and $\left\{X_{3} ; X_{4}\right\}$ for $C$, respectively. The redundancy between the combinations $\left\{X_{1} ; X_{2}\right\}$ and $\left\{X_{3} ; X_{4}\right\}$ is given by say, $0.5^{*}\left(r^{2}\left(X_{1}, X_{3}\right)+r^{2}\left(X_{2}, X_{4}\right)\right)$ and existence of strong LD between $X_{1}$ and $X_{3}$ and between $X_{2}$ and $X_{4}$ will result in similar measures of KWII association for both sets, making one of the sets statistically redundant.

\section{Stage I: Single-nucleotide polymorphism (SNP)-combination search strategy}

Let $S$ be the set of all genetic (SNPs) and environmental (non-genetic) variables (e.g., sex) and $C$ be the variable denoting the disease phenotype. The information theoretic metric $K W I I\left(X_{1} ; \ldots ; X_{k} ; C\right)$ is a measure of the association of the set of variables with the disease phenotype variable $C$ (i.e., how well they explain the disease phenotype). Using this metric and a redundancy measure, we iteratively search for combinations of variables up to a fixed number (say $\tau$ ) of iterations. Let the number of variables (except $C$ ) in a combination be defined as the "order" of the combination. In our method, we limit our search to up to second-order (or two-variable) combinations (i.e., we consider only $\left\{X_{i} ; C\right\}$ and $\left\{X_{i} ; X_{j} ; C\right\}$ combinations). Let $\theta$ be the set of variables and $\xi$ be the set of associated combinations output by our search method. Initially, both $\theta$ and $\xi$ are empty. In iteration $=1$, the variable $X_{k}$ having highest $\operatorname{KWII}\left(X_{k} ; C\right)$ is selected; thus $\theta=\left\{X_{k}\right\}$ and $\xi=\left\{\left(X_{k} ; C\right)\right\}$. Also $X_{k}$ is removed from $S$. In a subsequent iteration $=i$ ( $i>1)$, a new variable $X_{j} \in S$ is considered for selection and its single variable and two-variable combinations are formed and KWII computed (using, Eq. (2)) with variables already selected in the previous iterations. At the same time each of the combinations formed are checked for redundancy with combinations already in $\xi$ and of same order (using Eq. (3) and redundancy exceeding a threshold of 0.7). For example in iteration $=2$, for $X_{j} \in S$, the combinations $\left\{X_{j} ; C\right\}$ and $\left\{X_{k} ; X_{j} ; C\right\}$ are formed and $\left\{X_{j} ; C\right\}$ is checked for redundancy with $\left\{X_{k} ; C\right\}$. From all the new variables, the variable that has maximum KWII and all nonredundant combinations is selected. A variable with a redundant combination is dropped from consideration (i.e., removed from $S$ ) in subsequent iterations. Given the computational burden of determining redundancy with combinations of variables already selected, our selection procedure stops after a maximum of $\tau=50$ iterations. Thus, up to 50 variables with non-redundant combinations and highest KWII are selected. This stage yields a number of single and two-variable combinations and their KWII values, which are input to the second stage.

\section{Stage II}

We conduct logistic regression analysis on the one- and two-variable combinations obtained by our information theoretic search using the methods outlined by Cordell [7] and the significance of each combination is determined. The full single-locus model is

$$
\log (r /(1-r))=\mu+a x+d z
$$

where $r$ is the probability of each individual being a case, $\mu$ corresponds to the mean effect, the terms $a$ and $d$ correspond to the additive and dominance coefficient effects of the tested SNP variable, $x$ and $z$ are dummy variables with $x=1, z=-0.5$ for one homozygote genotype (AA), $x=0, z=0.5$ for the heterozygote genotypes $(A a)$, and $x=-1, z=-0.5$ for the other 
homozygote type ( $a a)$. The chi-square is used to compare the full single-locus model with the null model given by 0 values for both $a$ and $d$.

For SNP $\times$ SNP interactions, fully saturated model following Cordell's notation [7] is

$$
\log \left(\frac{r}{1-r}\right)=\mu+a_{1} x_{1}+d_{1} z_{1}+a_{2} x_{2}+d_{2} z_{2}+i_{a a} x_{1} x_{2}+i_{a d} x_{1} z_{2}+i_{d a} z_{1} x_{2}+i_{d d} z_{1} z_{2}
$$

where $r$ and $\mu$ are same as in Eq. (3), the terms $a_{1}, d_{1}, a_{2}$, and $d_{2}$ are the dominance and additive effect coefficients of the two SNPs, $i_{a a}, i_{a d}, i_{d a}$ and $i_{d d}$ represent their interaction coefficients, and $x_{i}$ and $z_{i}$ are dummy variables with $x_{i}=1, z_{i}=-0.5$ for one homozygous genotype ( $A A$ or $B B$ ), $x_{i}=0, z_{i}=0.5$ for the heterozygous genotypes $(A a$ or $B b)$, and $x_{i}=-1, z_{i}=-0.5$ for the other homozygous genotype ( $a a$ or $b b$ ). An interaction is tested by the deviance of the full two-locus model from the model minus the interaction terms with chi-square test.

\section{Data}

We have followed a candidate-gene-based approach and selected SNPs belonging to the candidate genes/regions in Table 1 for exploring both gene $\times$ RA and gene $\times$ gene $\times$ RA interactions using our two-stage approach. The start and end base-pair positions of each gene are obtained from http://www.pharmgkb.org/. Using the genes/regions from Table 1, we created the following three data sets for analysis:

- 7087 SNPs selected for analysis using all genes/ regions (Data Set 1)

- 5385 SNPs selected using all genes/regions except those that belong to only $6 \mathrm{p} 21.3$ and not to any other gene (Data Set 2)

- 3263 SNPs selected using genes not on chromosome 6 (Data Set 3)

Additionally, sex of the subjects and RA status were present in each data set as the environmental variable and the phenotype variable $(C)$.

\section{Results}

We have obtained many single-variable and two-variable interactions with the disease phenotype, only the combinations with high values of KWII are presented in Tables 2, 3, 4. The SNPs shown to be in genomic regions 6 p21.3 and 6 q23 do not overlap with any other gene. We found no interaction between the covariates sex and RA. Table 2 shows the single-variable combinations with $K W I I$ values greater than or equal to $95^{\text {th }}$ percentile of all the single-variable KWII obtained using our method for the respective data sets. Tables 3 and 4
Table I: Candidate genes, associated genes/regions and number of SNPs (\#s) in each

\begin{tabular}{|c|c|c|}
\hline Gene & Chr & No. SNPs \\
\hline TNFRSFIB & I & 17 \\
\hline PADI4 & 1 & 8 \\
\hline PTPN22 & 1 & 10 \\
\hline FCRL3 & I & 10 \\
\hline FCGR3A & I & 3 \\
\hline FCGR3B & I & 4 \\
\hline ILIO & 1 & 6 \\
\hline ILIA & 2 & 3 \\
\hline$I L I B$ & 2 & 9 \\
\hline ITGAV & 2 & 27 \\
\hline STAT4 & 2 & 2 \\
\hline CTLA4 & 2 & 5 \\
\hline BTLA & 3 & 2 \\
\hline IL3 & 5 & 2 \\
\hline SLC22A4 & 5 & 15 \\
\hline ILI3 & 5 & 4 \\
\hline IL4 & 5 & 5 \\
\hline HAVCRI & 5 & II \\
\hline $6 p 21.3$ & 6 & 1702 \\
\hline MICA & 6 & 230 \\
\hline HLA-C & 6 & 20 \\
\hline NFKBILI & 6 & II \\
\hline LTA & 6 & 6 \\
\hline TNF & 6 & 5 \\
\hline HLA-DR & 6 & 21 \\
\hline VEGFA & 6 & 6 \\
\hline $6 q 23$ & 6 & $|84|$ \\
\hline OLIG3 & 6 & 2 \\
\hline TNFAIP3 & 6 & 5 \\
\hline IL6 & 7 & 2 \\
\hline IRF5 & 7 & 4 \\
\hline C5 & 9 & 8 \\
\hline DLG5 & 10 & 20 \\
\hline MS4AI & 11 & 12 \\
\hline MHC2TA & 16 & 7 \\
\hline CARDI5 & 16 & 10 \\
\hline RUNXI & 21 & 3044 \\
\hline MIF & 22 & 12 \\
\hline
\end{tabular}

show the two-variable combinations with KWII values greater than or equal to $95^{\text {th }}$ percentile of all the two variable KWII obtained using our method for the respective data sets. The $95^{\text {th }}$ percentile value for each data set is reported with each table where $K W I I 95_{j}^{i}$ denote the $95^{\text {th }}$ percentile KWII for combinations of order $i$ and data set $j$. Additionally, to assess the overall strength of the KWII values we have obtained, we have calculated the KWII values of each single-variable combination for all 7088 variables, and 50,000 twovariable combinations randomly chosen from the list of $25,116,328$ pairs of variables. The $95^{\text {th }}$ percentile of these were found to be $K W I I 95_{\text {overall }}^{1}=0.01$ (onevariable combinations) and KWII95 $5_{\text {overall }}^{2}=0.004$ (twovariable combinations). All interactions reported in Tables 2, 3, 4 have KWII higher than these values. We have detected several one-variable associations in 6p21.3, HLA-DR, and RUNX1 (Table 2) and also in 
Table 2: $\{S N P ; C\}$ interactions with KWII values $\geq 95^{\text {th }}$ percentile of the I-variable KWII obtained for Data Set I, Data Set 2, Data Set 3

\begin{tabular}{|c|c|c|c|}
\hline SNP & Gene/genome region & KWII & $p-$ Value $^{b}$ \\
\hline \multicolumn{4}{|c|}{ Data Set I: KWII95 $5_{1}^{1}=0.015^{a}$} \\
\hline$r s 2395175$ & HLA-DR & 0.195 & 0 \\
\hline rs660895 & $6 p 21.3$ & 0.189 & 0 \\
\hline rs691007l & $6 p 21.3$ & 0.163 & 0 \\
\hline rs3763312 & 6p21.3 & 0.151 & 0 \\
\hline \multicolumn{4}{|c|}{ Data Set 2: KWII951 $=0.075$} \\
\hline rs2395175 & HLA-DR & 0.195 & 0 \\
\hline rs7192 & HLA-DR & 0.094 & 0 \\
\hline rs312987| & HLA-DR & 0.079 & 0 \\
\hline rs3129882 & HLA-DR & 0.075 & 0 \\
\hline \multicolumn{4}{|c|}{ Data Set 3: KWII95 $5_{2}^{1}=0.02$} \\
\hline rs731059 & RUNXI & 0.048 & 0 \\
\hline rs $475 \mid 42$ & RUNXI & 0.024 & $1.3 \times 10^{-11}$ \\
\hline
\end{tabular}

${ }^{\text {a }} K W I I 95_{j}^{i}$ denotes the $95^{\text {th }}$ percentile $K W I I$ for combinations of order $i$ and Data Set $j$.

${ }^{b} p$-Value obtained using logistic regression

Table 3: $\left\{S N P_{1} ; S N P_{2} ; C\right\}$ interaction with $K W I I$ values $\geq 95^{\text {th }}$ percentile of the two variable KWII obtained for Data Set I and consisting of SNPs only in 6p21.3 (and not in any candidate gene), for Data Set 2, and for Data Set 3)

\begin{tabular}{|c|c|c|c|}
\hline SNPI-SNP2 & Gene/genome region I - Gene/genome region 2 & KWII & $p-$ Value $^{\mathrm{b}}$ \\
\hline \multicolumn{4}{|c|}{ Data Set I: KWIIS $5_{1}^{2}=0.07^{\mathrm{a}}$} \\
\hline rs2647050-rs2858332 & 6p21.3-6p21.3 & 0.144 & 0 \\
\hline rs9357|52-rs2858332 & 6p21.3-6p21.3 & 0.098 & 0 \\
\hline rs9275|4|-rs285833| & $6 p 21.3-6 p 21.3$ & 0.092 & 0 \\
\hline rs7774434-rs2856718 & $6 p 21.3-6 p 21.3$ & 0.08 & 0 \\
\hline rs927537I-rs7765379 & $6 p 21.3-6 p 21.3$ & 0.076 & $3.35 \times 10^{-14}$ \\
\hline rs660895-rs7755224 & $6 p 21.3-6 p 21.3$ & 0.073 & $1.73 \times 10^{-7}$ \\
\hline rs9357|52-rs9275555 & $6 p 21.3-6 p 21.3$ & 0.07 & $9.98 \times 10^{-8}$ \\
\hline \multicolumn{4}{|c|}{ Data Set 2: KWII95 $2=0.02$} \\
\hline rs926387I-rs9263969 & MICA-MICA & 0.03 & $1.35 \times 10^{-8}$ \\
\hline rsII967684-rs2523608 & MICA-MICA & 0.025 & $2.22 \times 10^{-14}$ \\
\hline rs926387I-rs259650I & MICA-MICA & 0.022 & $1.81 \times 10^{-5}$ \\
\hline rsl| $967684-r s 7755852$ & MICA-MICA & 0.02 & $2.45 \times 10^{-8}$ \\
\hline rs3873380-rs7755852 & MICA-MICA & 0.02 & $1.39 \times 10^{-9}$ \\
\hline \multicolumn{4}{|c|}{ Data Set 3: KWII95 ${ }_{3}^{2}=0.01$} \\
\hline rsI542876-rs1513737 & $R U N X I-R U N X I$ & 0.015 & $1.85 \times 10^{-6}$ \\
\hline
\end{tabular}

${ }^{\text {a }} K W I I 95_{j}^{i}$ denotes the $95^{\text {th }}$ percentile $K W I I$ for combinations of order $i$ and Data Set $j$.

${ }^{\mathrm{b}} \mathrm{p}$-Value obtained using logistic regression.

Table 4: The two-variable interactions with KWII values $\geq 95^{\text {th }}$ percentile $(0.01)$ obtained using SNPs on gene $\times$ gene pairs on Data Set I

\begin{tabular}{|c|c|c|c|}
\hline SNPI-SNP2 & Gene/genome region I - Gene/genome region 2 & KWII & $p-V a l u e^{a}$ \\
\hline rs9275596-rsI542876 & 6p21.3-RUNXI & 0.01914 & $2.05 \times 10^{-6}$ \\
\hline rs $2856725-r s \mid 542876$ & $6 \mathrm{p} 21.3-R U N X I$ & 0.01743 & $3.61 \times 10^{-5}$ \\
\hline rs9275596-rs1041778 & $6 \mathrm{p} 21.3-R U N X I$ & 0.01471 & $8.25 \times 10^{-7}$ \\
\hline rs7770216-rs563495 & $6 p 21.3-6 q 23$ & 0.01444 & $4.15 \times 10^{-7}$ \\
\hline rs7755852-rs 2745443 & $6 p 21.3-6 q 23$ & 0.01275 & $6.22 \times 10^{-7}$ \\
\hline rs9275698-rs| 883468 & $6 p 21.3-6 q 23$ & 0.01262 & $4.51 \times 10^{-7}$ \\
\hline rs4673260-rs I 2190331 & CTLA4-6q23 & 0.01385 & $7.86 \times 10^{-8}$ \\
\hline rs|206684-rs651084 & $6 \mathrm{q} 23-R U N X I$ & 0.01261 & $6.12 \times 10^{-5}$ \\
\hline rs2844729-rs16984549 & 6p2I.3-RUNXI & 0.01255 & $5.71 \times 10^{-7}$ \\
\hline rs2856725-rs104I778 & 6p21.3-RUNXI & 0.01247 & $6.54 \times 10^{-6}$ \\
\hline
\end{tabular}

${ }^{a} p$-Value obtained using logistic regression. 


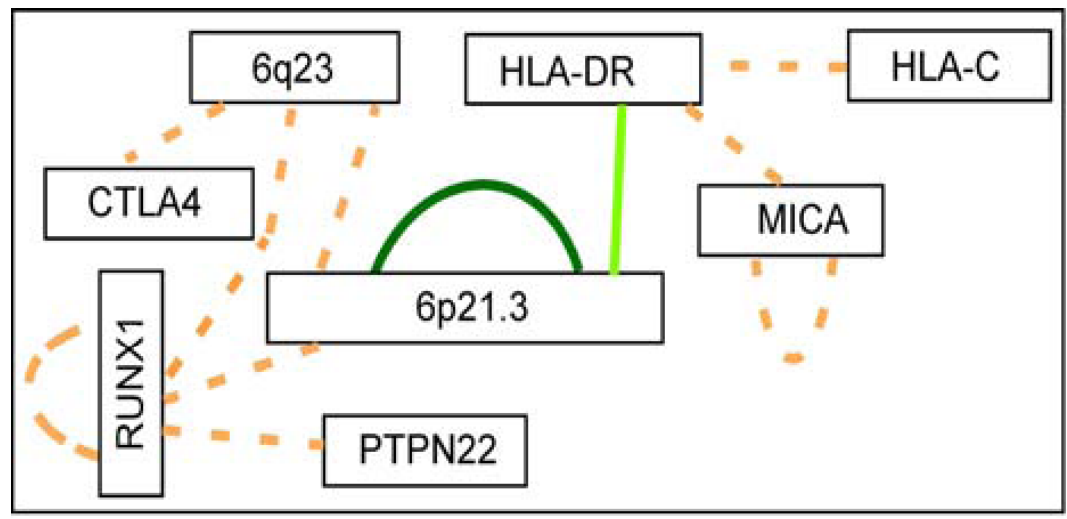

\title{
Gene x Gene Interactions obtained.
}

\author{
- Kwii>0.1 (Strong) \\ - $0.1>$ Kwii $>0.05$ (Moderate) \\ - Kwii<0.05 (Weak) \\ solid line: $p$-value $<10^{-15}$ \\ dotted line: $10^{-15} \leq p$-value $\leq 10^{-4}$
}

Figure I

Gene $\times$ gene interactions obtained.

6q23 and HLA-C (with $K W I I 95_{\text {overall }}^{1}<K W I I<95^{\text {th }}$ percentile for respective data sets, not shown in tables). The strongest of the two-variable interactions are from the SNPs in region 6p21.3 (Table 3) obtained using Data Set 1 . We have created the other two data sets because it was felt that several relatively weaker interactions are difficult to detect in the presence of the strongest interactions in 6p21.3. Using Data Sets 2 and 3, we found several two-variable KWII in genes MICA and RUNX1 (Table 3). Also several two-variable interactions are detected among SNPs in HLA-C,HLA-DR, MICA, and $6 \mathrm{p} 21.3$ (with $K W I I 95_{\text {overall }}^{2}<K W I I<95^{\text {th }}$ percentile for respective data sets, not shown in tables). Also we observed an interaction between rs11811771 (PTPN22) with rs2828104 (RUNX1) with $p$-value $2.7 \times 10^{-5}$ and $K W I I=0.095$. Separately we also calculated KWII for two-SNP combinations for Data Set 1 wherein the SNPs belong to different genes/genomic regions (Table 4).

The KWII values of two-variable combinations greater than $K W I I 95_{\text {overall }}^{2}=0.004$ are used to construct the gene $\times$ gene interaction diagram (Figure 1$)$. We have categorized these interactions as: 1$)$ strong $(K W I I \geq 0.1)$ in green, 2$)$ moderate $(0.1>K W I I \geq 0.05$ in light green, and 3 ) weak in orange. Also, bold lines indicate $p$-values $<10^{-15}$ while dotted lines denote $10^{-15} \leq p$-value $\leq 10^{-4}$.

\section{Discussion}

We have used a two-stage strategy to search for single SNPs and SNP $\times$ SNP interactions associated with RA. Using our analysis on the candidate genes, we have found several strong interactions on 6p21.3 and interactions among SNPs on genes previously reported to be related with RA and other autoimmune diseases. For example, RUNX1 has been reported to be associated with systemic lupus erythematosus and psoriasis (two autoimmune diseases) [8,9] while associations of region 6q23 and MICA with RA has been reported by Thomson et al. [10] and Martinez et al. [11], respectively. Detecting genes and environmental factors interacting to increase the susceptibility to disease risk is a very challenging task for many reasons, particularly for the large size of the data and presence of confounding factors such as LD, presence of phenocopies, locus heterogeneity, and population stratification. Information theoretic methods have high power in detecting gene $\times$ gene interactions and have the advantage of being simpler and computationally faster; KWII-based interaction analysis has been employed in $[3,4]$. Also, our method can be used when the genetic and environmental variables have different numbers of classes or when the phenotype has more than two classes. Although we initially planned for a genome-wide analysis, given the large size of the data, we were able to execute only a few iterations using our computational resources. Therefore, we decided to follow a candidate-gene-based approach. We believe that with the help of additional hardware, it is possible to implement our search strategy in a distributed computing environment employing multiple processors and to explore many more interactions with moderate to low magnitudes that are potentially associated with RA.

\section{List of abbreviations used}

GAW16: Genetic Analysis Workshop 16; KWII: $k$-way interaction information; LD: Linkage disequilibrium; RA: Rheumatoid arthritis; SNP: Single-nucleotide polymorphism.

\section{Competing interests}

The authors declare that they have no competing interests.

\section{Authors' contributions}

PC developed the computational methods and carried out the statistical genetics analysis. AZ was involved in 
the development of the computational analysis. LS participated in the statistical genetics analysis and interpretations. MR conceived the study and was involved all aspects of design and coordination.

\section{Acknowledgements}

The Genetic Analysis Workshops are supported by NIH grant ROI GM03 I575 from the National Institute of General Medical Sciences.

This article has been published as part of BMC Proceedings Volume 3 Supplement 7, 2009: Genetic Analysis Workshop 16. The full contents of the supplement are available online at http://www.biomedcentral.com/ $\underline{1753-656 \mid / 3 \text { ? issue }=\text { S7. }}$.

\section{References}

I. Liu Z and Lin S: Multilocus LD measure and tagging SNP selection with generalized mutual information. Genet Epidemiol 2005, 29:353-364.

2. Moore JH, Gilbert JC, Tsai CT, Chiang FT, Holden T, Barney N and White BC: A flexible computational framework for detecting, characterizing, and interpreting statistical patterns of epistasis in genetic studies of human disease susceptibility. J Theoret Biol 2006, 24I:252-26I.

3. Chanda P, Sucheston L, Zhang A, Brazeau D, Freudenheim JL, Ambrosone $C$ and Ramanathan M: AMBIENCE: a novel approach and efficient algorithm for identifying informative genetic and environmental interactions associated with complex phenotypes. Genetics 2008, 180:1191-1210.

4. Chanda P, Zhang A, Brazeau D, Sucheston L, Freudenheim JL, Ambrosone $C$ and Ramanathan M: Information-theoretic metrics for visualizing gene-environment interactions. $\mathrm{Am} \mathrm{J}$ Hum Genet 2007, 81:939-963.

5. Shannon CE: A mathematical theory of communication. Bell Sys Tech J 1948, 27:379-423, 623-656.

6. Jakulin A: Machine learning based on attribute interactions. [Ph.D. Thesis] Ljubljana, Slovenia, Department of Computer Science, University of Ljubljana; 2005.

7. Cordell HJ: Epistasis: what it means, what it doesn't mean, and statistical methods to detect it in humans. Hum Mol Genet 2002, I I:2463-2468.

8. Alarcon-Riquelme ME: A RUNX trio with a taste for autoimmunity. Nat Genet 2003, 35:299-300.

9. Yamada R, Tokuhiro S, Chang X and Yamamoto K: SLC22A4 and RUNXI: identification of RA susceptible genes. J Mol Med 2004, 82:558-564.

10. Thomson W, Barton A, Ke X, Eyre S, Hinks A, Bowes J, Donn R, Symmons D, Hider S, Bruce IN, Wellcome Trust Case Control Consortium, Wilson AG, Marinou I, Morgan A, Emery P, YEAR Consortium, Carter A, Steer S, Hocking L, Reid DM, Wordsworth P, Harrison P, Strachan D and Worthington J: Rheumatoid arthritis association at 6q23. Nat Genet 2007, 39:|43|-|433.

II. Martinez A, Fernandez-Arquero M, Balsa A, Rubio A, Alves $\mathrm{H}$, Pascual-Salcedo D, Martin-Mola E and de la Concha EG: Primary association of a MICA allele with protection against rheumatoid arthritis. Arthritis Rheum 200I, 44:|26|-1265.
Publish with Bio Med Central and every scientist can read your work free of charge

"BioMed Central will be the most significant development for disseminating the results of biomedical research in our lifetime. "

Sir Paul Nurse, Cancer Research UK

Your research papers will be:

- available free of charge to the entire biomedical community

- peer reviewed and published immediately upon acceptance

- cited in PubMed and archived on PubMed Central

- yours - you keep the copyright
BioMedcentral 